\title{
Pressure-induced superconductivity and modification of Fermi surface in type-II Weyl semimetal $\mathrm{NbIrTe}_{4}$
}

\author{
Qing-Ge Mu $\mathbb{B D}^{1 凶}$, Feng-Ren Fan ${ }^{1}$, Horst Borrmann ${ }^{1}$, Walter Schnelle ${ }^{1}$, Yan Sun ${ }^{1}$, Claudia Felser (iD) and Sergey Medvedev (iD ${ }^{1 凶}$
}

Weyl semimetals (WSMs) hosting Weyl points (WPs) with different chiralities attract great interest as an object to study chiralityrelated physical properties, topological phase transitions, and topological superconductivity. Quantum oscillation measurements and theoretical calculations imply that the type-II WPs in $\mathrm{NblrTe}_{4}$ are robust against the shift of chemical potential making it a good material for pressure studies on topological properties. Here we report the results of electrical transport property measurements and Raman spectroscopy studies under pressures up to $65.5 \mathrm{GPa}$ accompanied by theoretical electronic structure calculations. Hall resistivity data reveal an electronic transition indicated by a change of the charge carrier from multiband character to hole-type at $\sim 12 \mathrm{GPa}$, in agreement with the calculated Fermi surface. An onset of superconducting transition is observed at pressures above $39 \mathrm{GPa}$, with critical temperature increasing as pressure increases. Moreover, theoretical calculations indicate that WPs persist up to highly reduced unit cell volume $(-17 \%)$, manifesting that $\mathrm{NblrTe}_{4}$ is a candidate of topological superconductor.

npj Quantum Materials (2021)6:55; https://doi.org/10.1038/s41535-021-00357-y

\section{INTRODUCTION}

Recently, Weyl semimetals (WSMs) have attracted extensive attention in condensed matter physics ${ }^{1-4}$. Intensive studies were performed focusing on exploring new WSMs, topological phase transition, and topological superconductors ${ }^{1-7}$. From materials aspect, WSMs generally require the breaking of time reversal symmetry or inversion symmetry ${ }^{1,5,6}$. Theoretically, WSMs can be classified into two types. In type-I WSMs, the Fermi surface shrinks to nondegenerate Weyl points (WPs) with conserved Lorentz invariance ${ }^{5,6}$. In contrast, in type-II WSMs, the WPs appear at the contact of electron and hole pockets, which breaks Lorentz invariance ${ }^{5,6}$. WPs always come in pairs with opposite chiralities. Many exotic physical properties related to this kind of band crossing were observed in WSMs, such as the chiral anomaly, Fermi arcs, large magnetoresistance, and anomalous Hall effect ${ }^{4,8-14}$. Also, they constitute a platform for the detection of axion quasiparticles and may host unconventional superconductivity ${ }^{15-18}$.

The variation of the thermodynamic parameter pressure is a powerful method to modify the Fermi surface of a solid and to tune its carrier density without inducing chemical impurities. The monopnictides $\mathrm{TaPn}$ and $\mathrm{NbPn}(P n=\mathrm{P}, \mathrm{As})$ were predicted to be type-I WSMs ${ }^{6}$. Subsequently, quantum oscillation and angleresolved photoemission spectroscopy (ARPES) studies confirmed the exotic band structure at ambient pressure $e^{8-11,13}$. Moreover, high-pressure studies on transport properties and superconductivity were performed ${ }^{7,19-22}$. Interestingly, pressure induces a phase transition at $\sim 14 \mathrm{GPa}$ in TaAs, and the new phase was suggested to exhibit Weyl fermions different from the ambient pressure phase 7 . However, in contrast to other topological materials, only TaP displays superconducting transition at an extremely high pressure of $\sim 70 \mathrm{GPa}$, which is accompanied by a structural transition ${ }^{20}$. TaAs shows tip-induced superconductivity in the point contact spectroscopy ${ }^{23}$. The transition metal dichalcogenides $\mathrm{MoTe}_{2}, \mathrm{WTe}_{2}$, and the ternary compounds $M M^{\prime \prime}$ $\mathrm{Te}_{4}\left(M=\mathrm{Nb}, \mathrm{Ta}, M^{\prime}=\mathrm{Ir}\right.$, Rh) were proposed to be type-II WSMs, and some of them were experimentally confirmed ${ }^{12,24-30}$. High pressure-driven superconductivity following the dramatic suppression of the large magnetoresistance was reported for $\mathrm{WTe}_{2}$ and TalrTe ${ }_{4}{ }^{15,16,31}$. However, the topological nature of the superconducting state remains an open question under pressure.

$\mathrm{Nb} \mathrm{Te}_{4}$ features an orthorhombic lattice structure without inversion-symmetry, which is isostructural to TalrTe $\mathrm{T}_{4}$ and can be considered as a ternary variant of $\mathrm{WTe}_{2}{ }^{32}$. Recently, ARPES studies uncovered two different Fermi arc connections on opposite termination of (001) crystal faces ${ }^{33}$. Quantum oscillation measurements were performed by three different groups ${ }^{34-36}$. The magnetoresistance exhibits large values, and no saturation was observed up to a field of $35 \mathrm{~T}^{34-36}$. However, Hall measurements reveal that samples of $\mathrm{NblrTe}_{4}$ can adopt different Fermi levels, indicating that its Fermi surface is very sensitive to changes of the chemical potential, while the WPs persist against the shift of Fermi level as suggested from calculations ${ }^{34-36}$. These features make $\mathrm{NblrTe}_{4}$ a good platform to study the effect of pressure on its topological properties.

Here, we report the effects of high pressure on electrical transport properties and crystal structure of the type-II WSM $\mathrm{Nb}^{2} \mathrm{re}_{4}$ under external pressures up to $65.5 \mathrm{GPa}$. Hall effect measurements reveal that the Fermi surface changes from a multiband character to hole dominated at $\sim 12 \mathrm{GPa}$. Superconductivity is detected at pressures above $39 \mathrm{GPa}$, and the onset critical temperature $T_{\mathrm{c}}$ increases monotonically with further compression. The observed pressure-induced superconductivity in $\mathrm{NblrTe}_{4}$ is in good agreement with the recent results for the isostructural compound TalrTe ${ }_{4}{ }^{31}$, demonstrating that the superconductivity under compression is an intrinsic property of this class of compounds.

\section{RESULTS}

\section{Crystal structure and transport property measurements at} ambient pressure

$\mathrm{NblrTe}_{4}$ crystalizes in an orthorhombic crystal structure without inversion symmetry and exhibits a characteristic layer stacking along the $c$-axis (Fig. 1a) ${ }^{32}$. Along $a$-axis, $\mathrm{Nb}$ and Ir atoms each can

\footnotetext{
${ }^{1}$ Max Planck Institute for Chemical Physics of Solids, Dresden, Germany. ${ }^{\circledR}$ email: qingge.mu@cpfs.mpg.de; sergiy.medvediev@cpfs.mpg.de
} 

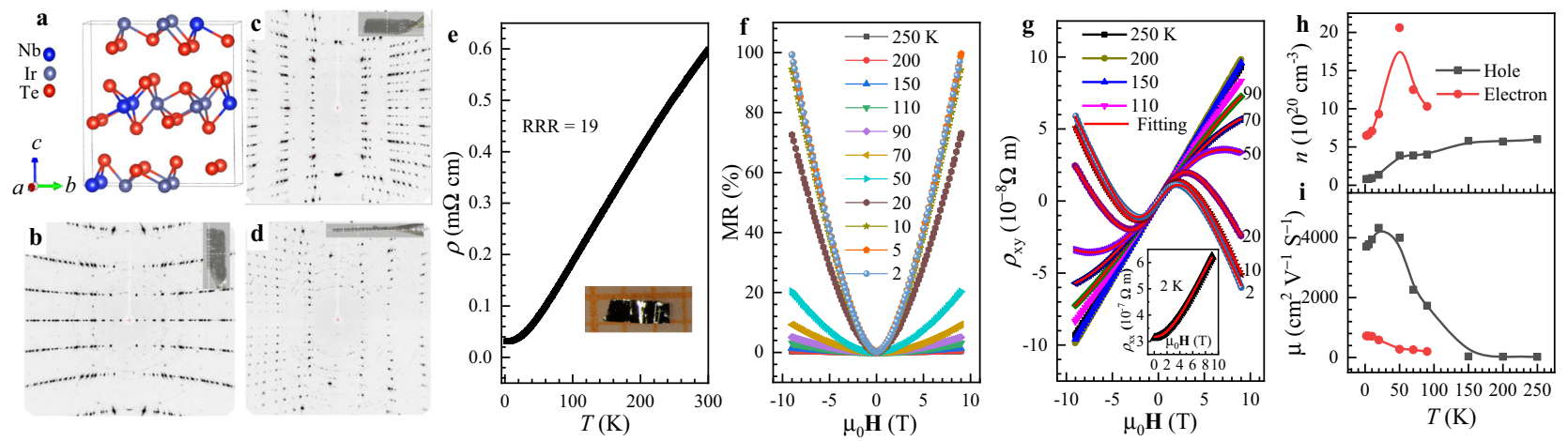

Fig. 1 Crystal structure and transport properties at ambient pressure. a The schematic crystal structure of NbIrTe . b-d The SXRD patterns on $a$-axis, $b$-axis, and $c$-axis. The insets are the optical picture of corresponding direction. e Resistivity $\rho(T)$ at ambient pressure. f, $\mathbf{g}$ Magnetoresistance $\mathrm{MR}(\mathbf{B})$ and Hall resistivity $\rho_{\mathrm{xy}}(\mathbf{B})$ at selected temperatures. The inset shows the magneto-resistivity $\rho_{\mathrm{xx}}(\mathbf{B})$ curve at $2 \mathrm{~K}$. The red solid lines represent the fitting according to two-band model. $\mathbf{h}, \mathbf{i}$ The extracted carrier density and mobility with respect to temperature.

be viewed as chain structures of edge-sharing octahedral units $\mathrm{NbTe}_{6}$ or $\operatorname{IrTe}_{6}$. The chains extend alternately along $b$-axis forming an individual layer, and the sequence is Nb-Ir...Ir-Nb. Figure $1 \mathrm{~b}-\mathrm{d}$ show the single crystal $x$-ray diffraction (SXRD) patterns characterizing different crystallographic axis at room temperature, and the insets are the corresponding optical pictures of the long plate-like crystal. The as-grown orthorhombic crystals are easily cleaved perpendicular to the $c$-axis, and the longer edge of the obtained $a b$-plane is the crystallographic $a$-axis. The clean diffraction pattern and the small width of the reflections confirm the good crystal quality. The space group is $P m n 2_{1}$, and the refined lattice parameters are $a=3.7805(5) \AA, b=12.5146(11) \AA$, and $c=13.1140$ (13) $\AA$, which are consistent with previous results ${ }^{32}$. We note that extra weak spots are detected on the diffraction pattern of $a$-axis, and the amount is less than $1 \%$. After carefully matching with possible impurity phases, we propose that these extra diffraction spots suggest a modulation of the crystal structure.

We performed electrical resistivity, magnetoresistance, and Hall resistivity measurements at ambient pressure, in which the current was along $a$-axis, and the magnetic field was perpendicular to the $a b$-plane. The data are shown in Fig. 1. The inset of Fig. $1 \mathrm{e}$ is a photo of the actual crystal used for measurements under ambient pressure and high pressure. The resistivity is $0.6 \mathrm{~m} \mathrm{~cm}$ at room temperature and exhibits metallic behavior down to $1.8 \mathrm{~K}$ (Fig. 1e) with a residual resistivity ratio (RRR) of 19. These specific values are comparable with previous reports ${ }^{34-36}$. The magnetic field dependence of magnetoresistance $M R(B)=(\rho$ (B) $-\rho(0)) / \rho(0) \times 100 \%$, is shown in Fig. 1f. All MR(B) curves follow a power law behavior $\sim \mathbf{B}^{\alpha}$ without the tendency of saturation, and $a$ changes from 1.5 to 1.9 as temperature increases to $250 \mathrm{~K}$. The same value of 1.5 was reported in TalrTe 4 at $1.7 \mathrm{~K}^{37}$. The $\mathrm{MR}(9 \mathrm{~T})$ at $2 \mathrm{~K}$ is $100 \%$.

Hall resistivity curves as a function of magnetic field $\rho_{\text {xy }}(\mathbf{B})$ at selected temperatures are shown in Fig. 1g. For $T \leq 90 \mathrm{~K}$, the $\rho_{\mathrm{xy}}(\mathbf{B})$ varies nonlinear, indicating that both electrons and holes contribute to the electrical transport properties in this temperature range. The sign of the slope at $2 \mathrm{~K}$ changes from positive to negative as magnetic field increases, as reported in ref. ${ }^{36}$. According to two-band model, electrons are the majority carriers, and adopt low mobility, while the minority hole-type carriers have high mobility. For $T \geq 110 \mathrm{~K}$, the curves exhibit linear magnetic field dependence with positive curvature, suggesting that holes become the dominant carriers. The Hall coefficient $R_{\mathrm{H}}\left(R_{\mathrm{H}}=\rho_{\mathrm{xy}} / \mathrm{B}\right)$ in linear $\rho_{\text {xy }}(\mathbf{B})$ gives the one-band carrier density based on $n_{\mathrm{h}}=1$ / $\mathrm{e} R_{\mathrm{H}}$, and the mobility is derived from $\mu=1 /$ ne $\rho$, while the nonlinear Hall resistivity is described by two-band model:

$$
\begin{gathered}
\rho_{\mathrm{xy}}=\frac{\mathbf{B}}{e} \frac{\left(\mu_{\mathrm{h}}^{2} n_{\mathrm{h}}-\mu_{\mathrm{e}}^{2} n_{\mathrm{e}}\right)+\left(\mu_{\mathrm{h}} \mu_{\mathrm{e}}\right)^{2} \mathbf{B}^{2}\left(n_{\mathrm{h}}-n_{\mathrm{e}}\right)}{\left(\mu_{\mathrm{h}} n_{\mathrm{h}}+\mu_{\mathrm{e}} n_{\mathrm{e}}\right)^{2}+\left(\mu_{\mathrm{h}} \mu_{\mathrm{e}}\right)^{2} \mathbf{B}^{2}\left(n_{\mathrm{h}}-n_{\mathrm{e}}\right)^{2}} \\
\rho_{\mathrm{xx}}=\frac{1}{e} \frac{\left(n_{\mathrm{h}} \mu_{\mathrm{h}}+n_{\mathrm{e}} \mu_{\mathrm{e}}\right)+\left(n_{\mathrm{h}} \mu_{\mathrm{e}}+n_{\mathrm{e}} \mu_{\mathrm{h}}\right) \mu_{\mathrm{e}} \mu_{\mathrm{h}} \mathbf{B}^{2}}{\left(\mu_{\mathrm{h}} n_{\mathrm{h}}+\mu_{\mathrm{e}} n_{\mathrm{e}}\right)^{2}+\left(\mu_{\mathrm{h}} \mu_{\mathrm{e}}\right)^{2} \mathbf{B}^{2}\left(n_{\mathrm{h}}-n_{\mathrm{e}}\right)^{2}}
\end{gathered}
$$

where $n_{\mathrm{h}}\left(n_{\mathrm{e}}\right)$ and $\mu_{\mathrm{h}}\left(\mu_{\mathrm{e}}\right)$ are the concentration and mobility of holes (electrons). These parameters are constrained with experimental resistivity $\rho_{\mathrm{xx}}$ according to Eq. (2). The obtained carrier density and mobility from the fit of $\rho_{x y}(\mathbf{B})$ are displayed in Fig. $1 \mathrm{~h}$, $\mathrm{i}$, and the inset of Fig. $1 \mathrm{~g}$ is the corresponding fit of $\rho_{\mathrm{xx}}(\mathbf{B})$ at $2 \mathrm{~K}$. Both fits give similar values of these parameters. As temperature increases, the carrier density of holes increases sharply at first, and then almost keeps constant. The mobility varies slightly, followed with continuous decrease. For the electrons, the concentration reaches a maximum at $\sim 50 \mathrm{~K}$, and the mobility keeps decreasing. Considering that the measured samples of different authors were grown with similar methods, they exhibit obvious differences in Hall resistivity. This suggests a drastic sensitivity of the Fermi surface to the chemical potential due to some unintentional doping. The shift of Fermi level, except for the variation of mobility ${ }^{36}$, is possibly the origin for the evolution of Hall resistivity with respect to temperature in $\mathrm{NbIrTe}_{4}$ at ambient pressure.

\section{Pressure-induced superconductivity}

The results of pressure dependent studies on electrical resistivity are presented on Fig. 2. The data (Fig. 2a, b) at pressures up to 40.6 GPa were collected on sample 1 using a diamond anvil cell (DAC) with $500 \mu \mathrm{m}$ culet. The sample demonstrates_similar to the ambient pressure data-metallic behavior upon cooling down to $1.8 \mathrm{~K}$. Interestingly, the pressure dependence of electrical resistivity is nonmonotonic. Upon applying pressure, the room temperature resistivity decreases to a minimum as pressure approaches to $\sim 12 \mathrm{GPa}$ and turns to increase as pressure is further increased. Finally, the resistivity decreases continuously as pressure is above $\sim 28 \mathrm{GPa}$. Electrical resistivity of $\mathrm{NblrTe}_{4}$ was further studied at higher pressures up to $65.5 \mathrm{GPa}$ by employing a DAC adopting a smaller culet $300 \mu \mathrm{m}$ (see Fig. 2c). At pressures $>39 \mathrm{GPa}$, drops of resistivity are detected at low temperature, indicating the onset of a superconducting transition. Electrical resistivity in normal state displays metallic behavior similar to that at lower pressures. For demonstrating the superconducting transition clearly, the temperature dependence of resistivity at low temperature is normalized to the value at $5 \mathrm{~K}$ (see Fig. $2 \mathrm{~d}$, the inset is the expanded view at $39.1 \mathrm{GPa}$ displaying a weak drop below $2 \mathrm{~K}$ ). With further 

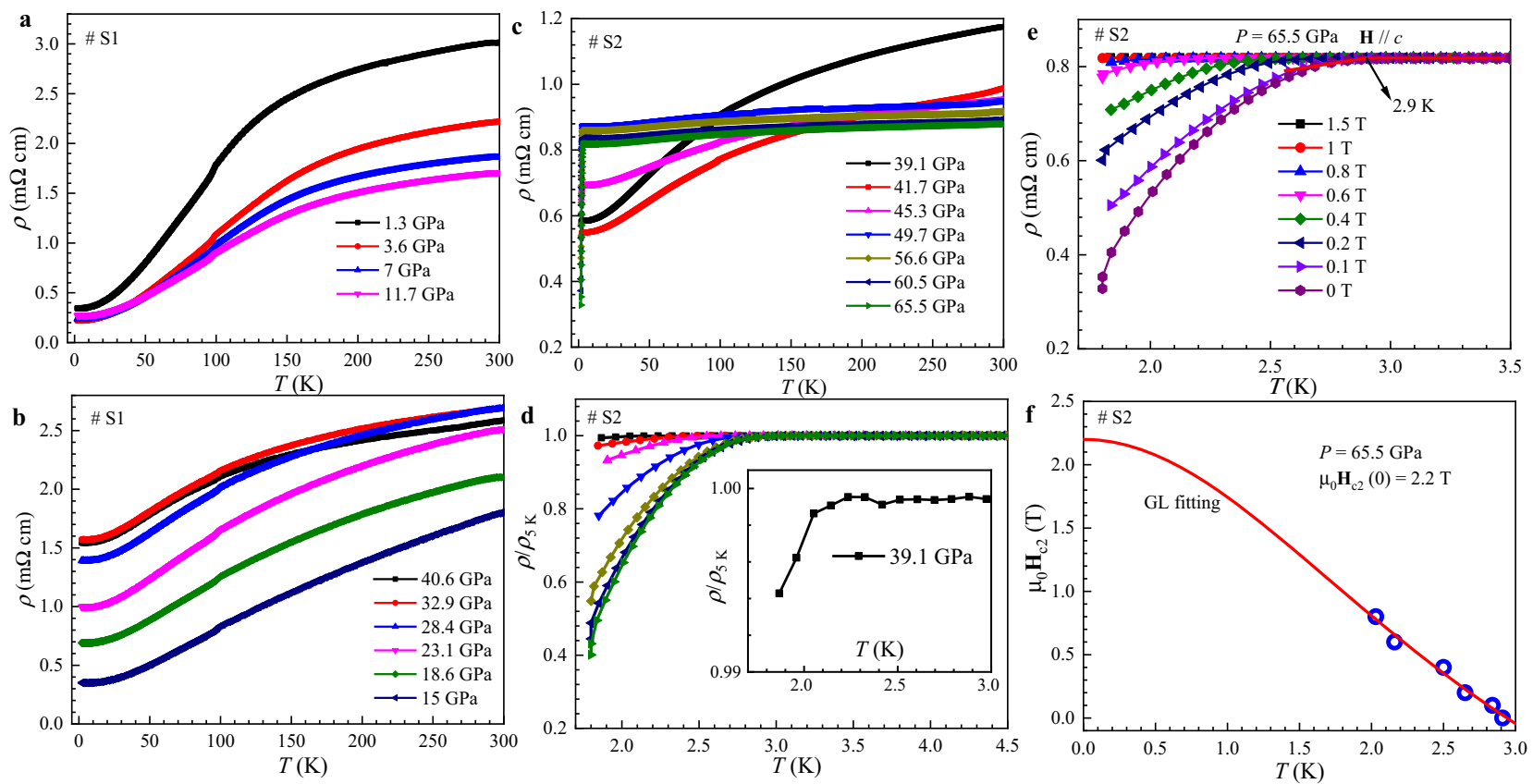

Fig. 2 Pressure-induced superconductivity. a, b $\rho(T)$ curves collected on sample 1 under pressures up to $40.6 \mathrm{GPa}$. c Pressure-induced superconductivity under higher pressures above $39 \mathrm{GPa}$. d Normalized $\rho(T)$ around superconducting transition, and the inset is the expanded view at $39.1 \mathrm{GPa}$. e, f Upper critical magnetic field measurements with field along the c-axis under pressure $65.5 \mathrm{GPa}$. e Superconducting transition under different magnetic fields up to $1.5 \mathrm{~T}$. $\mathbf{f}$ The obtained upper critical field depicted as a function of temperature. The red line is G-L fitting curve and blue circles are experimental data.
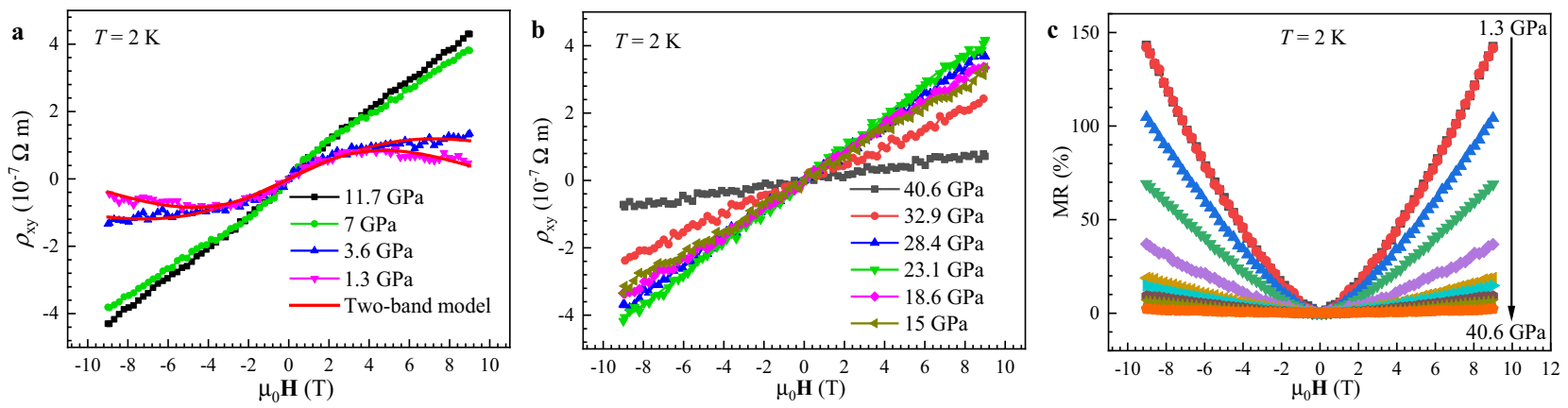

Fig. 3 Pressure effects on Hall resistivity and magnetoresistance. a Nonlinear magnetic field dependence of Hall resistivity $\rho_{\text {xy }}(\mathbf{B})$ with pressures below $11.7 \mathrm{GPa}$. The red lines are fittings according to two-band model. $\mathbf{b}$ Linear $\rho_{\mathrm{xy}}(\mathbf{B})$ curves with pressures up to $40.6 \mathrm{GPa}$. $\mathbf{c}$ The magnetoresistance $\mathrm{MR}(\mathrm{B})$ under pressures up to $40.6 \mathrm{GPa}$.

compression, the drop becomes more obvious, and the onset $T_{\mathrm{c}}$ is slightly increasing to $2.9 \mathrm{~K}$ at $65.5 \mathrm{GPa}$. However, the superconducting transition is broad and no zero-resistivity state is achieved in the applied pressure range. Higher pressure is required to further investigate the pressure-induced superconductivity.

The pressure-induced superconductivity in $\mathrm{NblrTe}_{4}$ crystals was confirmed by measuring the resistivity $\rho(T)$ under various static magnetic fields up to $1.5 \mathrm{~T}$ at $65.5 \mathrm{GPa}$ applied along the $c$-axis (Fig. 2e). With magnetic field increase, the onset $T_{c}$ shifts to lower temperature, and the drop of resistivity lifts. No superconducting transition can be detected above $1.8 \mathrm{~K}$ when magnetic field increases to $1.5 \mathrm{~T}$. The onset $T_{\mathrm{c}}$ (for zero field marked with an arrow in Fig. 2e) is determined by the temperature where the resistivity deviates from normal resistivity. The corresponding upper critical fields are plotted as a function of temperature and shown in Fig. 2f. According to Ginzburg-Landau theory, $H_{\mathrm{c} 2}(T)=$ $H_{\mathrm{c} 2}(0)\left(1-t^{2}\right) /\left(1+t^{2}\right)$ with $t=T / T_{\mathrm{c}}$, the upper critical field at zero temperature $\mu_{0} H_{\mathrm{c} 2}(0)$ is estimated to be $2.2 \mathrm{~T}$, which is larger than the value $\sim 0.87 \mathrm{~T}$ from $\mathrm{TalrTe}_{4}{ }^{31}$. It is noted that this is much lower than the Pauli-Clogston limit $5.4 \mathrm{~T}$, which is calculated from $\mu_{0} H_{\mathrm{P}}$ $[\mathrm{T}]=1.84 T_{\mathrm{c}}[\mathrm{K}]$. The Ginzburg-Landau coherence length $\xi(0)$ is about $12 \mathrm{~nm}$, as calculated by $H_{\mathrm{c} 2}(0)=\Phi_{0} /\left(2 \pi \xi^{2}(0)\right)$, where $\Phi_{0}=$ $2.07 \times 10^{-15} \mathrm{~Wb}$ is the flux quantum.

\section{Pressure-driven modification of electronic structure}

To study pressure effects on electronic structure, we carried out Hall resistivity $\rho_{x y}(\mathbf{B})$ and magnetoresistance $\mathrm{MR}(\mathbf{B})$ measurements at pressures up to $40.6 \mathrm{GPa}$ (Fig. 3). At pressure of $1.3 \mathrm{GPa}$, the sign of the slope for $\rho_{\mathrm{xy}}(\mathbf{B})$ changes from positive to negative as magnetic field increases from 0 to $9 \mathrm{~T}$ (Fig. 3a) similar to the behavior at ambient pressure. This suggests that the multiband character of the Fermi surface remains, and the majority carriers are electrons with low mobility. The multiband feature survives up to $11.7 \mathrm{GPa}$ as indicated by the nonlinear $\rho_{x y}(\mathbf{B})$ curves (Fig. 3a). However, with further increase of pressure, the $\rho_{\text {xy }}(\mathbf{B})$ curves display a linear magnetic field dependence (Fig. 3b), indicating that holes are the main charge carriers. The slope of the linear $\rho_{\text {xy }}(\mathbf{B})$ curves becomes 

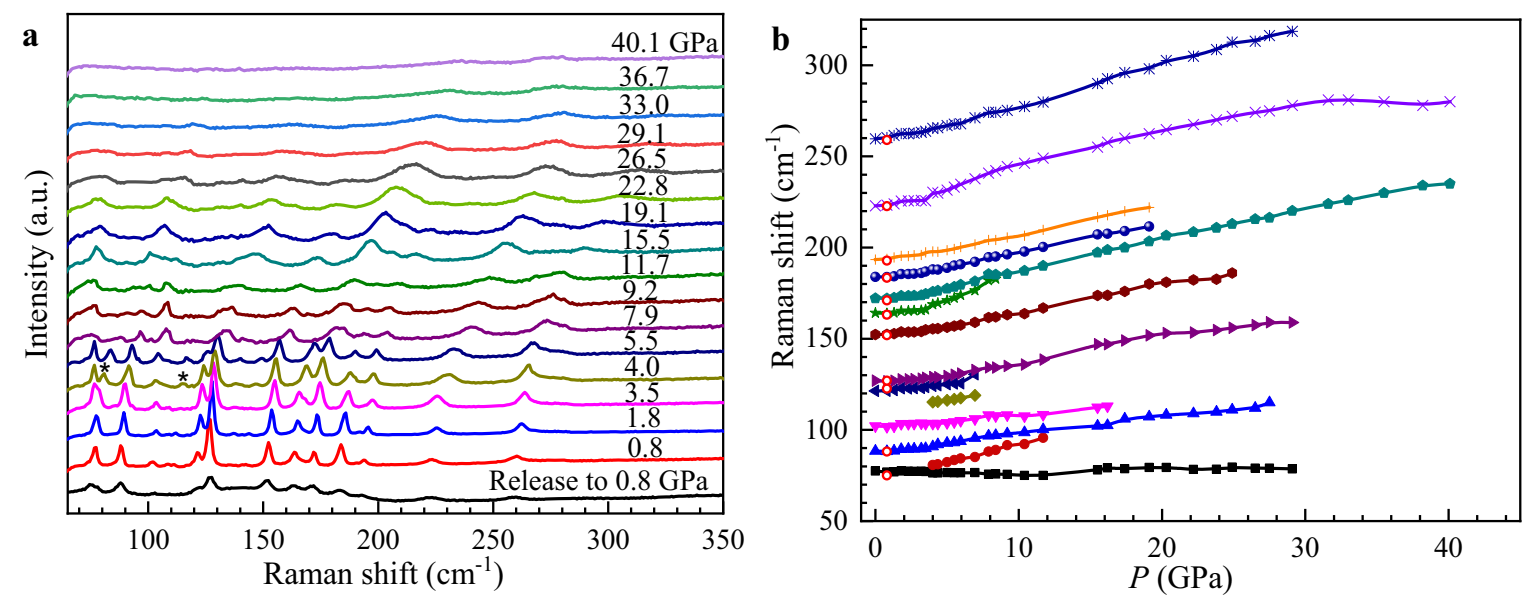

Fig. 4 Raman spectroscopy measurement under pressure. a Raman spectra with pressures up to $40.1 \mathrm{GPa}$, including the spectrum with pressure decrease to $0.8 \mathrm{GPa}$. The stars represent the new modes which appeared as pressure approached to $3.5 \mathrm{GPa}$. $\mathbf{b}$ Pressure dependence of Raman shifts. The open red circles are Raman shifts when pressure releases to $0.8 \mathrm{GPa}$.

larger when pressure approaches to $23.1 \mathrm{GPa}$, and is followed by a reduction upon further compressing, implying that the concentration of hole decreases slightly at first, and then increases. We did the same analysis of $\rho_{\mathrm{xy}}(\mathbf{B})$ as done at ambient pressure, and the extracted carrier density and mobility are summarized in the phase diagram. Similar to ambient pressure data, $\mathrm{MR}(\mathbf{B})$ exhibits a power law dependence $\sim \mathbf{B}^{a}$. The exponent $a$ is 1.4 below $11.7 \mathrm{GPa}$, and increases to 1.6 at pressure of $32.9 \mathrm{GPa}$ upon compression. These values are close to the value of 1.5 at ambient pressure.

\section{Raman spectroscopy studies}

We performed Raman spectroscopy study of $\mathrm{NblrTe}_{4}$ under pressures up to $40.1 \mathrm{GPa}$ to detect possible structural transitions in connection with the observed variations of the transport behavior at $\sim 12 \mathrm{GPa}$. Raman spectra under selected pressures are plotted in Fig. 4a. Both the incident laser (unpolarized) and backscattered laser beams were parallel to the $c$-axis. Based on group analysis, $23 \mathrm{~A}_{1}$ and $12 \mathrm{~A}_{2}$ optical modes can be detected with this backscattering geometry. Several obvious peaks were observed at $0.8 \mathrm{GPa}$, and the Raman shifts are in accordance with previous Raman measurements using polarized laser ${ }^{38}$. The pressure dependence of Raman shift is shown in Fig. 4b. Upon compressing, the Raman spectra show blue shift due to the shrinkage of lattice. Note that a shoulder peak appears near the peak with frequency $70 \mathrm{~cm}^{-1}$ under $3.5 \mathrm{GPa}$, and the peak becomes obvious under $4 \mathrm{GPa}$. The two newly emerging small peaks that can be recognized are marked by asterisks in Fig. 4a. In contrast to the total modification of the Raman spectrum at a major (reconstructive) structural transition, the observed profiles of the Raman spectra of $\mathrm{NblrTe}_{4}$ under different pressures remain the same. Additionally, in comparison with the spectrum of the isostructural TalrTe ${ }_{4}{ }^{39}$, more weak vibrational modes should be detected. Further considering that no structural transition was observed from synchrotron XRD in TalrTe ${ }_{4}{ }^{31}$. We suggest that a lattice distortion instead of major structural transition occurs at $\sim 3.5 \mathrm{GPa}$. In contrast, no anomaly is observed in Raman spectra and in the pressure coefficient of Raman shift at $\sim 12 \mathrm{GPa}$. Just the peak width increases, and the intensity decreases with pressure further increase due to nonhydrostatic pressure conditions at high pressure and the enhanced luminescence of the stressed diamond anvils. The vibrational peaks are too weak to be detected when pressure is above $40.1 \mathrm{GPa}$. As the pressure is released to $0.8 \mathrm{GPa}$ (Fig. 4a), the Raman spectrum is well consistent with the one at low pressure besides peaks have broadened due to the poorer sample quality after compression. Also, the observed Raman shifts are in good agreement with the data obtained upon compression (Fig. 4b). This indicates that the crystal structure of $\mathrm{NblrTe}_{4}$ is reversible with respect to pressure.

\section{Electronic structure calculations}

Using density-functional theory (DFT) calculations, we simulated the pressure effects on the electronic structure of $\mathrm{NblrTe}_{4}$. Since no major structural transition was detected from Raman spectroscopy, we compressed the lattice volume within the range of $\beta=\left(V-V_{0}\right) /$ $V_{0} \sim[-17 \%, 0]$ based on the experimental crystal structure above, where $\beta$ is shrinkage rate, and $V_{0}$ is the volume at ambient pressure. The band structure at ambient pressure is shown in Fig. 5a. Two hole bands (band 1 and 2) and two electron bands (band 3 and 4) cross the Fermi level doing contribution to the conductivity. Figure $5 b, c$ are the Fermi surfaces of band 1-4 with $\beta=0$ and $-17 \%$, respectively. In Fig. $5 b$, the Fermi surfaces of band 3 and 4 are much larger than the Fermi surfaces of band 1 and 2. In Fig. $5 c$, all the four Fermi surfaces shrink, but the Fermi surface of band 2 (with hole character) is much larger than the other three ones. As a consequence, holes dominate the transport behavior. Thus, comparing Fig. 5b, c, we can find the switch of the charge carrier character from electrons to holes, which is consistent with the above Hall effect measurements. Correspondingly, the density of states near Fermi level decreases slightly and then increases continuously with further compression (Fig. 5 d). $\mathrm{NblrTe}_{4}$ is a type-ll WSM $^{30}$, and we have also investigated the evolution of its WPs in momentum space. All the WPs locate in the $k_{z}=0$ plane slightly above the Fermi level, and in the $k_{\mathrm{z}}=0.21$ plane below Fermi level at ambient pressure. Upon compressing, such kinds of WPs exist all the way up to the maximum considered shrinkage $-17 \%$ (Table 1 ).

\section{DISCUSSION}

The obtained results demonstrate that applying pressure significantly modifies the Fermi surface of $\mathrm{NblrTe}_{4}$, and a superconducting transition is observed. According to DFT calculations, these WPs persist with the volume reduced by $17 \%$, indicating that $\mathrm{NblrTe}_{4}$ is promising to exhibit topological superconductivity.

Magnetoresistance curves $\mathrm{MR}(\mathbf{B})$ measured under high pressure follows the same power law behavior as at ambient pressure, and shows no hint of saturation. The amplitude at $9 \mathrm{~T}$ is suppressed with pressure increase, and it follows the evolution of mobility very well (Fig. 6a). However, the suppression of MR is not as sharp as 

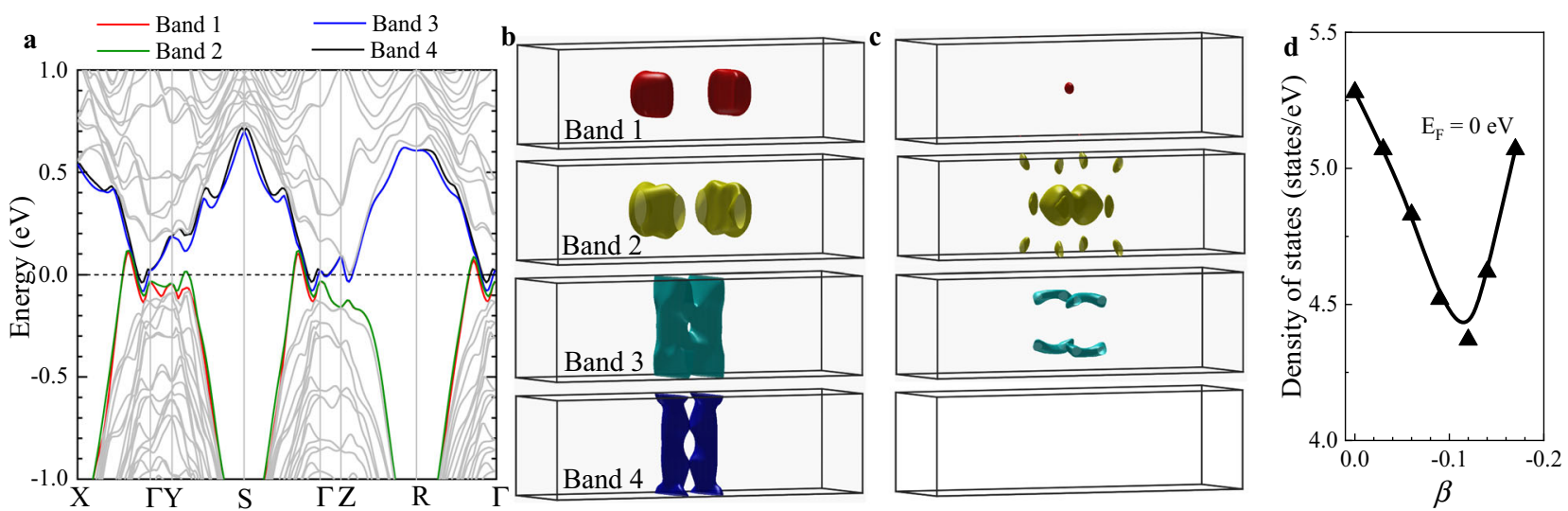

Fig. 5 DFT calculations. a The band structure at ambient pressure and the horizontal dashed line denotes the Fermi level. The compressing effects on Fermi surface of each band are demonstrated in b without compression, and c largest shrinkage of $-17 \%$. $\mathbf{b}$ The corresponding density of states at Fermi surface as a function of shrinking rate $\beta$, where $\beta=\left(V-V_{0}\right) / V_{0}$, and $V_{0}$ is the volume at ambient pressure. d The density of states at Fermi level upon compression.

\begin{tabular}{|c|c|c|c|c|c|}
\hline \multirow[t]{2}{*}{$\beta=\left(V-V_{0}\right) / V_{0}$} & \multicolumn{3}{|c|}{ Position } & \multirow[t]{2}{*}{ Energy (meV) } & \multirow[t]{2}{*}{ Chern number } \\
\hline & $k_{1} \frac{2 \pi}{a_{1}}$ & $k_{2}\left(\frac{2 \pi}{a_{2}}\right)$ & $k_{3}\left(\frac{2 \pi}{a_{3}}\right)$ & & \\
\hline \multirow[t]{2}{*}{0} & 0.14 & 0.20 & 0.00 & 118 & 1 \\
\hline & 0.04 & 0.17 & 0.21 & -74 & -1 \\
\hline \multirow[t]{2}{*}{$-3 \%$} & 0.13 & 0.24 & 0.00 & 102 & 1 \\
\hline & 0.06 & 0.24 & 0.20 & -45 & -1 \\
\hline \multirow[t]{2}{*}{$-6 \%$} & 0.12 & 0.28 & 0.00 & 82 & 1 \\
\hline & 0.07 & 0.29 & 0.20 & -14 & -1 \\
\hline \multirow[t]{2}{*}{$-9 \%$} & 0.11 & 0.34 & 0.00 & 59 & 1 \\
\hline & 0.08 & 0.21 & 0.34 & -27 & -1 \\
\hline \multirow[t]{2}{*}{$-12 \%$} & 0.09 & 0.40 & 0.00 & 13 & 1 \\
\hline & 0.07 & 0.23 & 0.38 & -26 & -1 \\
\hline \multirow[t]{2}{*}{$-15 \%$} & 0.10 & 0.42 & 0.00 & 19 & 1 \\
\hline & 0.07 & 0.29 & 0.42 & -13 & -1 \\
\hline \multirow[t]{2}{*}{$-17 \%$} & 0.05 & 0.14 & 0.00 & 54 & 1 \\
\hline & 0.03 & 0.40 & 0.25 & -32 & -1 \\
\hline
\end{tabular}

reported for other topological materials ${ }^{15,16,31}$, and the MR even exhibits a slight enhancement at low pressures. Pressure effects on resistivity and Hall resistivity are complex (Fig. 6b). Hall effect measurements reveal that the conduction behavior changes from two-type (with electron being the majority) to dominant hole-type at $\sim 12 \mathrm{GPa}$, and the carrier concentration decreases at first, and then increases continuously.

The resistivity shows nonmonotonic pressure dependence, and reaches a minimum at $\sim 12 \mathrm{GPa}$, the same pressure where hole concentration achieves a maximum. Such nonmonotonic pressure effects on resistivity were observed for topological insulator $\beta$ $\left.\mathrm{Bi}_{4}\right|_{4}$, topological material $\mathrm{ZrTe}_{5}$, and type-I semimetal TaP and $\mathrm{NbP}^{22,40,41}$. Such behavior may be explained by a possible topological phase transition with an energy gap opening at the crossing point, a pressure-induced competition of multiband or pressure-induced Lifshitz transition $22,40,41$. According to our DFT calculations, holes dominate the Fermi surface at a lattice compression by $17 \%$, giving rise to the experimentally observed variation of Hall resistivity and resistivity.
An onset of superconducting transition is observed below $2 \mathrm{~K}$ at 39.1 GPa where the hole concentration is much enhanced. The onset $T_{c}$ increases along with increase of pressure, as it is also observed in TalrTe ${ }_{4}{ }^{31}$. The modification of the Fermi surface as well as the increased carrier density plays a significant role in the pressure-induced superconductivity. With regard to the topological property, the persistence of WPs when the volume shrinks by up to $17 \%$ makes $\mathrm{NblrTe}_{4}$ a candidate of topological superconductivity. However, the relationship between superconductivity and topological property calls for further investigations. Note that no anomalies are detected on the pressure dependence of the resistivity at $\sim 3.5 \mathrm{GPa}$, the pressure at which the Raman spectra suggest a possible pressure-induced lattice distortion.

In conclusion, we performed electrical transport property characterization and Raman spectroscopy measurements on $\mathrm{NblrTe}_{4}$ single crystals at pressures up to $65.5 \mathrm{GPa}$. Superconducting transition is observed when pressure exceeds $39 \mathrm{GPa}$, and the onset $T_{\mathrm{c}}$ increases continuously along with pressure increase. $T_{\mathrm{c}}$ at the highest applied pressure is $2.9 \mathrm{~K}$. From the Hall resistivity characterization, the charge carrier changes from multiband character to hole-type at $\sim 12 \mathrm{GPa}$ due to modification of Fermi surface. According to DFT calculations, WPs survive with volume reduction up to $17 \%$, implying that $\mathrm{NblrTe}_{4}$ is a candidate for topological superconductor. Our studies on pressure effect on $\mathrm{NblrTe}_{4}$ provide a promising platform for investigating the relationship between topological properties and superconductivity.

\section{METHODS}

\section{Crystal growth}

The $\mathrm{NblrTe}_{4}$ single crystals were grown out of excess Te which was used as flux. The mixture of high purity Nb powder ( $99.99 \%$ Alfa Aesar), Ir powder (99.9+\% Alfa Aesar), and Te pieces (99.9999\% Alfa Aesar) with atomic ratio 1:1:12 was put into an alumina crucible and sealed in a quartz tube with $200 \mathrm{mbar}$ Ar inside. The sealed quartz tube was heated to $1273 \mathrm{~K}$ in $20 \mathrm{~h}$ and kept for $48 \mathrm{~h}$, followed by cooling down to $973 \mathrm{~K}$ at a rate of $1 \mathrm{~K} \mathrm{~h}^{-1}$. Finally, the excess Te flux was removed by centrifugation of the ampule at $973 \mathrm{~K}$, and long plate-like single crystals were harvested. The crystals are shining and exhibit good ductility. Fresh surface was obtained by cleaving many times with Scotch tape.

\section{Transport property measurements and crystal structure characterization}

The crystal structure at ambient pressure was characterized with SXRD at room temperature, and the transport properties at ambient pressure were collected on Quantum Designed PPMS-9 using low-frequency ac excitation and the standard four-probe method. For high-pressure studies, nonmagnetic 

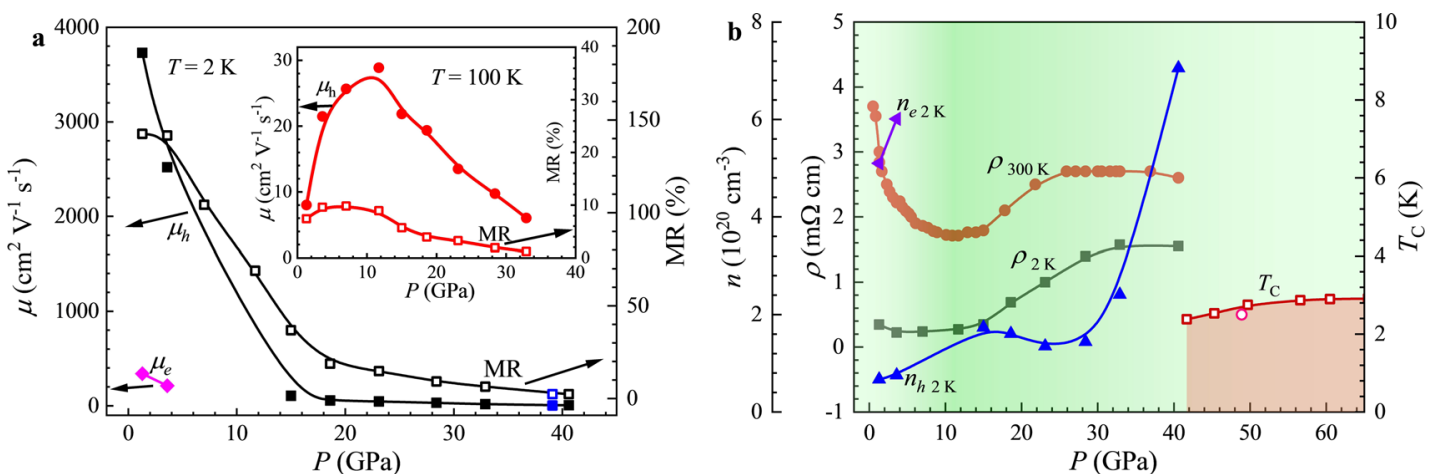

Fig. 6 Phase diagram. a Pressure effects on mobility and MR(9 T) at $2 \mathrm{~K}$, and the inset displays the data at $100 \mathrm{~K}$. b Phase diagram depicting pressure dependence of resistivity at 2 and $300 \mathrm{~K}$, carrier density at $2 \mathrm{~K}$ and the onset $T_{\mathrm{c}}$ from two samples.

DACs were used for Raman spectroscopy measurements and electrical transport property measurements. The DAC with culet $500 \mu \mathrm{m}$ and sample chamber $200 \mu \mathrm{m}$ was utilized to achieve pressures up to $50 \mathrm{GPa}$. For electrical transport measurements at higher pressures, the DAC with $300 \mu \mathrm{m}$ culet and $100 \mu \mathrm{m}$ sample chamber was used. The metallic gasket was made of $\mathrm{W}$ foil, and thin electrodes were produced by Pt foil. The sample and electrodes were insulated from the metallic gasket with a mixture of cubic BN and epoxy. $\mathrm{NaCl}$ was used as pressure transmitting medium to obtain quasihydrostatic conditions. Electrical resistivity and Hall resistivity measurements were performed with Van der Pauw method on the PPMS-9 at temperature down to $1.8 \mathrm{~K}$. The electric current was within the $a b$-plane, and the magnetic field was parallel to the $c$-axis. Raman spectra under pressures up to $40.1 \mathrm{GPa}$ were collected with a customary confocal micro-Raman spectrometer using unpolarized HeNe laser $(632.8 \mathrm{~nm})$ as the excitation source. The resolution of single-grating spectrograph is $1 \mathrm{~cm}^{-1}$.

\section{Theoretical calculations}

DFT calculations were performed using the Vienna ab initio simulation package $^{42,43}$, based on the projector augmented wave (PAW) method ${ }^{44}$. We have employed the Perdew-Burke-Ernzerhof (PBE) generalizedgradient approximation (GGA) function ${ }^{45}$. The adopted PAW-PBE pseudopotentials of $\mathrm{Nb}, \mathrm{Ir}$, and Te treat $4 p^{6} 4 d^{4} 5 s^{1}, 5 d^{8} 6 s^{1}$, and $5 s^{2} 5 p^{4}$ electrons as valence states. We set the energy cutoff as $400 \mathrm{eV}$, and used a $9 \times 3 \times 3$ Monkhorst-Pack k-point grid for atom-position optimizations. All the atoms were fully relaxed until the force on each of them was smaller than $0.01 \mathrm{eV}$ $\AA^{-1}$. Spin-orbit coupling was included for calculating Fermi surfaces and generating tight-binding Hamiltonian. The tight-binding model was built by means of maximally-localized Wannier functions (MLWFs) ${ }^{46-48}$. We set the Nb- $d$, Ir- $d$, and Te- $p$ orbitals as the initial wave functions for maximizing localization.

\section{DATA AVAILABILITY}

All relevant data are available from corresponding authors upon reasonable request.

Received: 4 February 2021; Accepted: 11 May 2021; Published online: 01 June 2021

\section{REFERENCES}

1. Wan, X., Turner, A. M., Vishwanath, A. \& Savrasov, S. Y. Topological semimetal and Fermi-arc surface states in the electronic structure of pyrochlore iridates. Phys. Rev. B 83, 205101 (2011).

2. Alicea, J. New directions in the pursuit of Majorana fermions in solid state systems. Rep. Prog. Phys. 75, 076501 (2012).

3. Armitage, N. P., Mele, E. J. \& Vishwanath, A. Weyl and Dirac semimetals in threedimensional solids. Rev. Mod. Phys. 90, 015001 (2018).

4. Yan, B. \& Felser, C. Topological materials: Weyl semimetals. Annu. Rev. Condens. Matter Phys. 8, 337-354 (2017).

5. Soluyanov, A. A. et al. Type-II Weyl semimetals. Nature 527, 495-498 (2015).
6. Weng, H., Fang, C., Fang, Z., Bernevig, B. A. \& Dai, X. Weyl semimetal phase in noncentrosymmetric transition-metal monophosphides. Phys. Rev. X 5, 011029 (2015).

7. Zhou, Y. et al. Pressure-induced new topological Weyl semimetal phase in TaAs. Phys. Rev. Lett. 117, 146402 (2016).

8. Huang, $X$. et al. Observation of the chiral-anomaly-induced negative magnetoresistance in 3D Weyl semimetal TaAs. Phys. Rev. X 5, 031023 (2015).

9. Shekhar, C. et al. Extremely large magnetoresistance and ultrahigh mobility in the topological Weyl semimetal candidate NbP. Nat. Phys. 11, 645-649 (2015).

10. Xu, S. Y. et al. Discovery of a Weyl fermion semimetal and topological Fermi arcs. Science 349, 613-617 (2015).

11. Xu, S. Y. et al. Discovery of a Weyl fermion state with Fermi arcs in niobium arsenide. Nat. Phys. 11, 748-754 (2015).

12. Huang, L. et al. Spectroscopic evidence for a type II Weyl semimetallic state in $\mathrm{MoTe}_{2}$. Nat. Mater. 15, 1155-1160 (2016).

13. $\mathrm{Xu}, \mathrm{N}$. et al. Observation of Weyl nodes and Fermi arcs in tantalum phosphide. Nat. Commun. 7, 11006 (2016).

14. Liang, T. et al. Anomalous Hall effect in $\mathrm{ZrTe}_{5}$. Nat. Phys. 14, 451-455 (2018).

15. Kang, D. et al. Superconductivity emerging from a suppressed large magnetoresistant state in tungsten ditelluride. Nat. Commun. 6, 7804 (2015).

16. Pan, X. C. et al. Pressure-driven dome-shaped superconductivity and electronic structural evolution in tungsten ditelluride. Nat. Commun. 6, 7805 (2015).

17. Qi, Y. et al. Superconductivity in Weyl semimetal candidate $\mathrm{MoTe}_{2}$. Nat. Commun. 7, 11038 (2016).

18. Gooth, J. et al. Axionic charge-density wave in the Weyl semimetal $\left(\mathrm{TaSe}_{4}\right)_{2} \mathrm{l}$. Nature 575, 315-319 (2019).

19. Einaga, M., Shimizu, K., Hu, J., Mao, Z. Q. \& Politano, A. Resistivity of Weyl semimetals NbP and TaP under pressure. Phys. Status Solidi RRL 11, 1700182 (2017).

20. Li, Y. et al. Concurrence of superconductivity and structure transition in Weyl semimetal TaP under pressure. npj Quant. Mater. 2, 66 (2017).

21. Guo, Z. et al. High-pressure phases of Weyl semimetals NbP, NbAs, TaP, and TaAs. Sci. China-Phys. Mech. Astron. 61, 038211 (2018).

22. Gupta, S. N. et al. Pressure-induced Lifshitz transition in NbP: Raman, x-ray diffraction, electrical transport, and density functional theory. Phys. Rev. B 97, 064102 (2018).

23. Wang, $\mathrm{H}$. et al. Discovery of tip induced unconventional superconductivity on Weyl semimetal. Sci. Bull. 62, 425-430 (2017).

24. Sun, Y., Wu, S. C., Ali, M. N., Felser, C. \& Yan, B. Prediction of Weyl semimetal in orthorhombic MoTe 2 . Phys. Rev. B 92, 161107 (2015).

25. Deng, K. et al. Experimental observation of topological Fermi arcs in type-II Weyl semimetal MoTe 2 . Nat. Phys. 12, 1105-1110 (2016).

26. Koepernik, K. et al. TalrTe 4 : a ternary type-II Weyl semimetal. Phys. Rev. B 93, 201101 (2016).

27. $\mathrm{Wu}, \mathrm{Y}$. et al. Observation of Fermi arcs in the type-II Weyl semimetal candidateWTe ${ }_{2}$. Phys. Rev. B 94, 121113 (2016).

28. Belopolski, I. et al. Signatures of a time-reversal symmetric Weyl semimetal with only four Weyl points. Nat. Commun. 8, 942 (2017).

29. Haubold, E. et al. Experimental realization of type-II Weyl state in noncentrosymmetric TalrTe 4 . Phys. Rev. B 95, 241108 (2017).

30. Li, L. et al. Ternary Weyl semimetal $\mathrm{NblrTe}_{4}$ proposed from first-principles calculation. Phys. Rev. B 96, 024106 (2017).

31. Cai, S. et al. Observation of superconductivity in the pressurized Weyl-semimetal candidate TalrTe . Phys. Rev. B 99, 020503 (2019). 
32. Mar, A. \& Ibers, J. A. Synthesis and physical-properties of the new layered ternary tellurides $\mathrm{MlrTe}_{4}(M=\mathrm{Nb}, \mathrm{Ta})$, and the structrue of $\mathrm{NblrTe}_{4}$. J. Solid State Chem. 97, 366-376 (1992)

33. Ekahana, S. A. et al. Topological Lifshitz transition of the intersurface Fermi-arc loop in $\mathrm{NblrTe}_{4}$. Phys. Rev. B 102, 085126 (2020).

34. Huang, X. W. et al. Magneto-transport and Shubnikov-de Haas oscillations in the type-Il Weyl semimetal candidate NblrTe ${ }_{4}$ flake. Chin. Phys. Lett. 36, 077101 (2019).

35. Schönemann, R. et al. Bulk Fermi surface of the Weyl type-Il semimetallic candidate $\mathrm{NblrTe}_{4}$. Phys. Rev. B 99, 195128 (2019).

36. Zhou, W. et al. Nonsaturating magnetoresistance and nontrivial band topology of type-II Weyl semimetal NblrTe 4 . Adv. Electron. Mater. 5, 1900250 (2019).

37. Khim, S. et al. Magnetotransport and de Haas-van Alphen measurements in the type-II Weyl semimetal TalrTe 4 . Phys. Rev. B 94, 165145 (2016).

38. Chen, $\mathrm{H}$. et al. Unusual polarization and temperature-dependent Raman response in Weyl semimetal $\mathrm{NblrTe}_{4}$. Solid State Commun. 289, 56-60 (2019).

39. Liu, Y. et al. Raman signatures of broken inversion symmetry and in-plane anisotropy in type-II Weyl semimetal candidate TalrTe 4 . Adv. Mater. 30, 1706402 (2018).

40. Zhou, Y. et al. Pressure-induced superconductivity in a three-dimensional topological material ZrTe 5 . Proc. Natl Acad. Sci. USA 113, 2904-2909 (2016).

41. Qi, Y. et al. Pressure-induced superconductivity and topological quantum phase transitions in a quasi-one-dimensional topological insulator: $\mathrm{Bi}_{4} \mathrm{I}_{4}$. npj Quant. Mater. 3, 4 (2018).

42. Kresse, G. \& Hafner, J. Ab initio molecular dynamics for open-shell transition metals. Phys. Rev. B Condens. Matter 48, 13115-13118 (1993).

43. Kresse, G. \& Furthmuller, J. Efficient iterative schemes for ab initio total-energy calculations using a plane-wave basis set. Phys. Rev. B 54, 11169-11186 (1996).

44. Blochl, P. E. Projector augmented-wave method. Phys. Rev. B Condens. Matter $\mathbf{5 0}$ 17953-17979 (1994).

45. Perdew, J. P., Burke, K. \& Ernzerhof, M. Generalized gradient approximation made simple. Phys. Rev. Lett. 77, 3865-3868 (1996).

46. Marzari, N. \& Vanderbilt, D. Maximally localized generalized Wannier functions for composite energy bands. Phys. Rev. B 56, 12847-12865 (1997).

47. Souza, I., Marzari, N. \& Vanderbilt, D. Maximally localized Wannier functions for entangled energy bands. Phys. Rev. B 65, 035109 (2001).

48. Mostofi, A. A. et al. wannier90: a tool for obtaining maximally-localised Wannier functions. Comput. Phys. Commun. 178, 685-699 (2008).

\section{ACKNOWLEDGEMENTS}

This work was financially supported by the ERC Advanced Grant no. 742068, "TOPMAT".

\section{AUTHOR CONTRIBUTIONS}

Q.-G.M. grew the crystals. Q.-G.M., S.M., and W.S. performed transport and Raman spectroscopy measurements. F.-R.F. and Y.S. performed DFT calculations. H.B. and Q.-G.M. carried out SXRD measurements. All authors discussed the results of the studies. Q.-G.M., S.M., F.-R.F., and W.S. co-wrote the paper. All authors commented on the manuscript.

\section{FUNDING}

Open Access funding enabled and organized by Projekt DEAL.

\section{COMPETING INTERESTS}

The authors declare no competing interests.

\section{ADDITIONAL INFORMATION}

Correspondence and requests for materials should be addressed to Q.-G.M. or S.M.

Reprints and permission information is available at http://www.nature.com/ reprints

Publisher's note Springer Nature remains neutral with regard to jurisdictional claims in published maps and institutional affiliations.

\begin{abstract}
(c) (i) Open Access This article is licensed under a Creative Commons adaptation, distribution and reproduction in any medium or format, as long as you give appropriate credit to the original author(s) and the source, provide a link to the Creative Commons license, and indicate if changes were made. The images or other third party material in this article are included in the article's Creative Commons license, unless indicated otherwise in a credit line to the material. If material is not included in the article's Creative Commons license and your intended use is not permitted by statutory regulation or exceeds the permitted use, you will need to obtain permission directly from the copyright holder. To view a copy of this license, visit http://creativecommons. org/licenses/by/4.0/.
\end{abstract}

(c) The Author(s) 2021 